\title{
Classical-classical transfer: CR interactions involving appetitive and aversive CSs and USs
}

\author{
MICHAEL J. SCAVIO, JR. \\ California State University, Fullerton, California 92634
}

\begin{abstract}
The present classical-classical transfer experiment involved three stages. In the first stage, tone-CS and water-US presentations were employed to condition the rabbit's jaw-movement response. In the second stage, the nictitating membrane response was conditioned using light-CS and shock-US pairings. In Stage 3, the tone and light stimuli were successively presented in a compound without USs with jaw-movement and nictitating membrane CRs being recorded. The results indicated that prior jaw-movement conditioning disrupted the initial acquisition of the nictitating membrane CR; compound tone and light presentations obliterated jaw-movement performance; and minimal cross-modal generalization of the nictitating membrane $\mathrm{CR}$ occurred to the tone on compound CS presentations. The findings were evaluated with regard to the manner in which Pavlovian mediation is produced in classical-instrumental transfer designs with the obtained results agreeing with opponent-process motivational interpretations of Pavlovian mediational effects.
\end{abstract}

The purpose of the present three-stage transfer experiment is to ascertain whether the compound presentation of two conditioned stimuli (CSs), originally paired with unconditioned stimuli (USs) with opposite hedonic properties, influences previously established conditioned response activity in the albino rabbit. Accordingly, in Stage 1, tone-CS and water-US pairings were given for jaw-movement (JM) conditioning and then, in Stage 2, light-CS and shock-US pairings were used for nictitating membrane (NM) conditioning. In the test stage, the tone and light CSs were successively presented in a compound without USs and JM and NM CRs were recorded.

A very popular position among learning theorists is that inherent classical conditioning operations within the instrumental paradigm produces a situational $\mathrm{CR}$ conditioned to a local CS. The situational CR is considered to exercise mediational control over instrumental behavior. However, there is theoretical disagreement with regard to the mechanism of mediation with associative (Logan \& Wagner, 1965; Spence, 1956; Trapold \& Overmier, 1972), incentive-motivational (Miller, 1963; Mowrer, 1960; Rescorla \& Solomon, 1967; Sheffield, 1966; Spence, 1956), and secondary drive/reinforcement (Hull, 1952; Mowrer, 1947) positions having been advanced.

To study the nature of Pavlovian mediation, several researchers (cf. Rescorla \& Solomon, 1967; Trapold \& Overmier, 1972) have utilized classical-instrumental transfer designs. In these experiments, separate stages of classical and instrumental conditioning precede a test stage in which the CS, previously established in the class-

This research was supported by USPHS grant MH-24518-01 and the Friends of the State University. The paper was sponsored by Russell Revlis who takes full editorial responsibility for its contents. ical stage, is presented during instrumental training with the usual result of a contingent change in the rate of instrumental responding. However, the data from classical-instrumental transfer designs may be reflecting the influence of mutually mediating CRs complicating the interpretation of Pavlovian mediational influences upon instrumental behavior. One of these CRs (the CS-elicited CR) is easily specified in that it has been previously established in the classical conditioning stage. The other CR is the situational CR conditioned to a local CS during instrumental training. Consequently, in the test stage of the classical-instrumental transfer experiment, interpolated presentations of the CS may result in mediational interaction between the CS-elicited CR and the situational CR. The effects produced by the $C R$ interactions may then govern instrumental performance. In support of the possibility of CR interactions, Scavio (1974) has found in a classical-classical transfer design that prior aversive NM conditioning interfered with subsequent appetitive JM conditioning to the same CS and that NM CR responding during JM conditioning decreases at a rate faster than extinction. The present work extends the study of CR interactions by investigating the effects of compounding two distinct CSs with separate reinforcement histories. This situation is analogous to the classical-instrumental transfer paradigm where the CS-elicited CR and the situational CR are evoked by separate but contiguously occurring CSs in the test stage.

\section{METHOD}

\section{Subjects}

The subjects were 24 male and female albino rabbits, 90-100 days old at the beginning of the experiment.

\section{Apparatus}

The rabbit preparations for NM and JM conditioning and the PDP-12 interface have been described elsewhere (Bissell \& 
Scavio, 1974; Scavio, 1974). The tone CS was a 500-msec $1,000-\mathrm{Hz} 82-\mathrm{dB}$ stimulus, while the light $\mathrm{CS}$ was a $500-\mathrm{msec}$ increase in illumination from $1 \mathrm{fc}$ to $4 \mathrm{fc}$. The US for JM conditioning was a 1-cc squirt of distilled water of 300-msec duration given directly into the oral cavity. The US for NM conditioning was a $50-\mathrm{msec} 4-\mathrm{mA} 60-\mathrm{Hz}$ electric shock delivered through electrodes embedded behind the left eye.

\section{Procedure}

All subjects were limited to $90 \mathrm{cc}$ of water each day during the experiment. On the 6 th day following the imposition of water deprivation, six rabbits were randomly assigned to each of four groups. Stage 1 training occupied the next 4 days, with Groups PP2 and PU2 receiving daily 30 paired presentations of the tone CS and water US for JM conditioning. Group UP2 received daily 30 unpaired presentations of tone and water, subject to the restrictions that in each block of 10 presentations an equal number of tone and water stimuli occur and no more than three successive presentations of the same stimulus occur. Group NP2 was placed in the conditioning chambers on each day for a period of time corresponding to the training sessions but received no stimuli. In Stage 2, lasting 3 days, Groups PP2, UP2, and NP2 received daily 80 light CS and shock US pairings for NM conditioning, while Group PU2 received daily 80 unpaired deliveries of light and shock subject to the order restrictions. In Stage 3, also lasting 3 days, Groups PP2, UP2, and NP2 received daily 60 presentations of the tone followed by the light. The time from tone offset to light onset was $500 \mathrm{msec}$. In addition, each session contained 20 test trials, occurring on every fourth trial, on which the light CS was presented alone. Group PU2 received 60 trials on each Stage 3 day in which the tone CS was presented alone so as to measure the course of JM CR extinction.

The CS-US interval on paired trials was $500 \mathrm{msec}$, and the intertrial intervals were randomized at values of 60,70 , and $80 \mathrm{sec}$ with a mean of $70 \mathrm{sec}$. The intertrial intervals for unpaired CS and US deliveries were randomized at values of 30 , 35 , and $40 \mathrm{sec}$ with a mean of $35 \mathrm{sec}$. Extensions of the NM and JM responses of $1 \mathrm{~mm}$ were considered to be CRs if they occurred during: the CS-US interval on paired trials, $500 \mathrm{msec}$ from CS onset on unpaired trials, a blank 500 -msec interval given to Group NP2 in Stage 1 at times corresponding to the recording intervals for Group PP2, or the tone and light CSs in Stage 3.

\section{RESULTS}

\section{Stage 1 JM Conditioning}

Groups PP2 and PU2 showed sustained daily acquisition of the JM CR reaching $70 \%$ on the final day. In contrast, Group UP2 indicated some pseudoconditioning reaching $38 \%$ on the last day while the response level of Group NP2 remained below 5\% on each day.

\section{Stage 2 NM Conditioning}

Groups UP2 and NP2 appeared to show initially faster acquisition of the NM CR compared to Group PP2. However, by the final day of training, all groups were responding near $85 \%$. Initial group differences were verified by an analysis of variance (ANOVA) conducted upon trials to the first CR (F = 8.79, $\mathrm{df}=2 / 15, \mathrm{p}<.01$ ), and a subsequent NewmanKeuls test showed that the mean trial value for Group PP2 $(X=128)$ was significantly greater $(\mathrm{ps}<.01)$ than the means for Groups UP2 $(X=63)$ and NP2 $(X=$ 68 ) with the latter two means not significantly different.

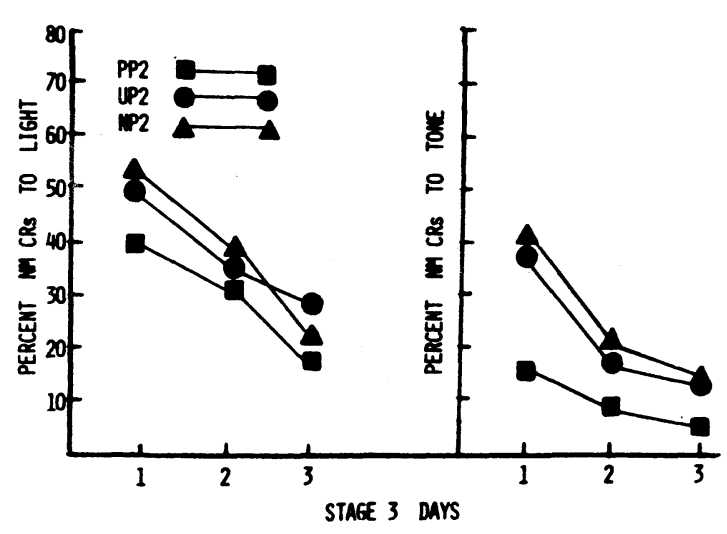

Figure 1. Mean percentage nictitating membrane CRs (NM $\mathrm{CRs}$ ) to the light CS plotted over the training days for groups in Stage 3 (left panel) and mean percentage generalized NM CRs to the tone CS plotted over training days for groups in Stage 3 (right panel).

NM CR percentages for Group PU2 did not rise above $4 \%$ on any day.

\section{Stage 3 NM and JM CR Performances}

The left panel of Figure 1 shows percent NM CRs elicited by the original light CS on test trials for Groups PP2, UP2, and NP2 on each Stage 3 day. This panel indicates similar group decrements in NM CR strength, and a subsequent ANOVA found no significant group differences. The right panel of Figure 1 illustrates the cross-modal generalization (CMG) of the NM CR to the tone CS on each day. CMG effects are most noticeable on Day 1 with Groups UP2 and NP2 exhibiting greater generalization than Group PP2 which had the tone as the CS for JM conditioning. An ANOVA conducted upon percent generalized NM CRs on Day 1 revealed significant group effects (F 3.93, df $=2 / 15, \mathrm{p}<.05)$, and a subsequent Newman-Keuls test indicated that generalized NM CR strength for Group PP2 (15\%) on Day 1 was significantly lower $(\mathrm{ps}<.05)$ than percentages recorded for Group UP2 (40\%) and NP2 (45\%) with the latter two means not significantly different.

Figure 2 shows percent JM CRs elicited by the tone CS element of the tone-light compound for Groups PP2, UP2, and NP2 on each Stage 3 day. Also provided is the extinction rate of the JM CR for Group PU2 which received only the tone $\mathrm{CS}$ in Stage 3 . The interesting feature of this panel is the immediate loss of JM CR responding in Group PP2 which had the tone as the CS in Stage $1 \mathrm{JM}$ conditioning. The performance of Group PP2 was characteristic of Groups UP2 and NP2, which received no Stage 1 JM conditioning. Furthermore, the higher extinction JM CR response rate shown by Group PU2 suggests that the inhibition of Group PP2's JM CR performance on tone-light compound trials can be traced to the use of the light as the CS for Stage 2 aversive NM conditioning. The magni- 


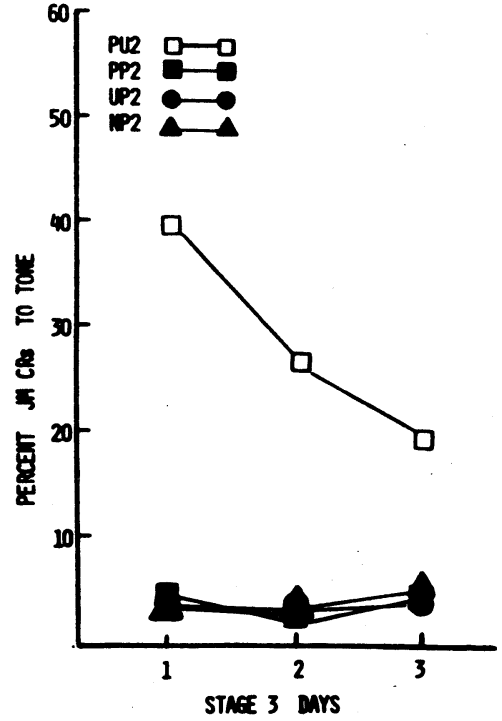

Figure 2. Mean percentage jaw-movement CRs (JM CRs) to the original tone CS plotted over Stage 3 training days for all groups.

tude of the JM CR levels between Groups PP2 and PU2 was so large as not to require statistical confirmation.

Cross-modal generalization phenomena were further investigated in an adjunct experiment using identical procedures except the modality of the CSs paired with USs was reversed. The results indicated that compound tone (used as the CS for Stage $1 \mathrm{NM}$ conditioning) and light (used as the CS for Stage $2 \mathrm{JM}$ conditioning) presentations in Stage 3 inhibited cross-modal generalization of the JM CR to the tone element. This was substantiated by an ANOVA conducted upon percent CMG responses on the initial block of 10 trials which located significant group effects $(F=3.99, \mathrm{df}=2 / 15$, $\mathrm{p}<.05)$. A companion Newman-Keuls test resulted in the mean generalization JM CR percentage for Group PP2 (10\%) being lower (ps <.05) than Group UP2's (52\%) and NP2's (60\%) with the latter two values not significantly different.

\section{DISCUSSION}

The data revealed that prior appetitive JM conditioning to a tone CS interfered with subsequent aversive NM conditioning to a light $C S$, compound stimulus presentations of tone and light disrupted JM CR performance, and minimal cross-modal generalization of the appetitive JM CR and aversive NM CR occurred to counterconditioned CS elements presented in a compound.

The Stage 3 results, showing the CMG inhibition of the light-elicited NM CR to the tone CS accompanied by decrements in JM CR levels, are exemplary evidence that the compounding of two CSs can produce $C R$ interactions. Accordingly, CR interactions may also be occurring in the test stage of classical-instrumental transfer designs where again two CSs, eliciting potentially mediating effects, occur. The unrecognized presence of such interactions could obscure the study of the Pavlovian mediational process. However, in classical-classical transfer designs, assessment of CR interaction effects is afforded with the possibility that the data may elucidate the mechanisms of mediation. Certain portions of the present data, along with previous demonstrations of CR interactions (Scavio, 1974), conform to deductions drawn from incentive-motivational views of Pavlovian mediation. These positions commonly hold that appetitive and aversive motivational systems have a reciprocally inhibitory relationship. Therefore, any attempt to arouse both systems simultaneously (as with concurrent CSs or USs) should lead to a mutual reduction in appetitive and aversive motivational strength. The findings of the blocking of the CMG of the NM CR to the appetitive CS at the same time an immediate loss in JM CR activity occurred, along with the interference shown in the initial phase of NM CR conditioning following JM CR conditioning, conform to an opponenet-process motivational interpretation. The latter finding may indicate that prior JM conditioning establishes situational stimuli as an appetitive CS complex which has the effect of disrupting momentarily NM CR formation.

The cross-modal generalization of the NM CR and the JM CR, although transitory, is especially interesting. Very little knowledge is available to Western researchers regarding the principles governing CMG. The present results seem to indicate that the reinforcement history of the cue serving as the CMG stimulus is an important consideration in the elaboration of generalized responding.

\section{REFERENCES}

Bissell, H. S., \& Scavio, M. J., JR. A minicomputer program for the resolution of response frequency and latency in classical conditioning preparations. Behavior Research Methods \& Instrumentation, 1974, 6, 439-442.

Huls, C. L. A behavior system. New Haven: Yale University Press, 1952.

Logan, F. A., \& WAgner, A. R. Reward and punishment. Boston: Allyn \& Bacon, 1965.

Mille R, N. E. Some reflections on the law of effect produce a new alternative to drive reduction. In $\mathbf{M}$. A. Jones (Ed.), Nebraska Symposium on Motivation, 1963. Lincoln: University of Nebraska Press, 1963.

Mowrer, O. H. On the dual nature of learning-a re-interpretation of "conditioning" and "problem solving." Harvard Educational Review, 1947, 17, 102-148.

MOWRER, O. H. Learning theory and behavior. New York: Wiley, 1960.

Rescorla, R. A., \& Solomon, R. L. Two process learning theory: Relationships between Pavlovian conditioning and instrumental learning. Psychological Review, 1967, 74, 151-182.

Scavio, M. J., JR. Classical-classical transfer: Effects of prior aversive conditioning upon appetitive conditioning in rabbits (Oryctolagus Cuniculus). Journal of Comparative \& Physiological Psychology, 1974, 86, 107-115.

Sheffield, F. D. New evidence on the drive-induction theory of reinforcement. In R. N. Haber (Ed.), Current research in motivation. New York: Rinehard \& Winston, 1966.

SPENCE, K. W. Behavior theory and conditioning. New Haven: Yale University Press, 1956.

Trapold, M. A., \& Overmier, J. B. The second learning process in instrumental conditioning. In A. H. Black \& W. F. Prokasy (Eds.), Classical conditioning II: Current theory and research. New York: Appleton-CenturyCrofts, 1972.

(Received for publication July 17, 1975.) 